\title{
Interface vitrectomy-a savior technique for vitreoretinal surgery
}

\begin{abstract}
Interface vitrectomy is a special vitrectomy technique based on performing the surgery at the interface between surface tension agents including air, liquid perfluorocarbon, or silicone oil and the retina. Interface vitrectomy technique allows good stabilization of the retina while preventing the increasing subretinal fluid and bleeding to interface during traction removal in vitreoretinal surgery. In this review, we aimed to summarize interface vitrectomy technique.
\end{abstract}

Keywords: interface vitrectomy, air, perfluorocarbon, silicon oil, assisted vitrectomy
Volume 9 Issue 4 - 2019

\author{
Burak Turgut,' Onur Çatak ${ }^{2}$ \\ 'Department of Ophthalmology, Faculty of Medicine, Onsekiz \\ Mart University, Turkey \\ ${ }^{2}$ Department of Ophthalmology, Faculty of Medicine, Firat \\ University, Turkey
}

Correspondence: Burak Turgut, Department of

Ophthalmology, Faculty of Medicine, Onsekiz Mart University,

Turkey,Tel+905337|28389, Email burakturgut@comu.edu.tr

Received: August 19,2019 | Published: August 30, 2019
Abbreviations: PFCL, perfluorocarbon liquid; SOIL, silicone oil; SRF, subretinal fluid; VRS, vitreoretinal surgery; ERM, epiretinal membrane; PVR, proliferative vitreoretinopathy; RD, retinal detachment; MVP, micro-vitrectomy probe; PFO, perfluoro-n-octane; IOL, Intraocular lens; RBs, retinal breaks

\section{Introduction}

Interface vitrectomy (IFV) is vitrectomy technique in which surgical processes are performed at the interface between surface tension agents such as intravitreal air, perfluorocarbon liquid (PFCL), silicone oil (SOIL) or substances immiscible in aqueous media such as vitreous humor, subretinal fluid (SRF), blood, fibrin and infusion fluid, and vitreoretinal tissue. ${ }^{1-6}$ The technique of IFV has been firstly described by Dr. Steve Charles. ${ }^{1-4}$ In IFV, scissors or forceps for vitreoretinal surgery (VRS), vitreous cutters and other VRS instruments are used outside SOIL, air, or PFCL bubble and at the interface between SOIL, air, or PFCL and residual vitreous, epiretinal membrane (ERM), and retina. IFV has been performed for removal of residual vitreous traction, retinectomy, ERM peeling/segmentation/ delamination, internal drainage of SRF, subretinal surgery, endo-laser photocoagulation, and diathermy. ${ }^{1-6}$

The main purposes of IFV are to enhance the visualization of the vitreous base and vitreoretinal pathologies and intraoperatively retinal stabilization whereby the interface between air, SOIL, and PFCLs and vitreoretinal tissue. ${ }^{1-5}$ IFV also prevents the increase of SRF during traction removal and bleeding to the interface during surgery. SOIL and air bubble float on infusion fluid, while PFCL sinks under all. The retina may be stabilized by the properties of spring dampening of air, by the viscous dampening of SOIL and inertial and gravitational dampening of PFCL ${ }^{1-9}$ IFV may be used under SOIL in re-operations with two-port infusion-free for recurrent retinal detachment (RD) due to proliferative vitreoretinopathy (PVR) or ERM under previously placed SOIL or in primary surgery following the stage of injection of air, SOIL, and PFCL. ${ }^{1-5}$ In this surgical technique, a microvitrectomy probe (MVP) is placed within the residual or peripheral vitreous with the port directed away from the retinal surface, and the vitreous removed. The technique is commonly used in vitrectomy of phakic cases. IN IFV, vacuum should not be applied when the MVP or soft-tip cannula is within the air, SOIL, or PFCL bubble. If not, the instrument may be locked with plugging, or the loss of SOIL or PFCL may occur. ${ }^{1-9} \mathrm{IFV}$ is a vitrectomy technique which its importance is not well-known. Thus, we aimed to summarize the properties and the main usage fields of IFV technique in this review.

\section{PFCL-assisted IFV}

PFCL has allows both inertial and gravitational stabilization with inertial and gravitational dampening effect to the retina for counteracting with dissection forces, unfolding retinal folds, and separating retina from vitreous. ${ }^{1}$ PFO (perfluoro-n-octane) is a PFCL which has a viscosity of approximately two-thirds that of the infusion fluid and a high specific gravity of about twice of its mass. Thus, PFO sinks and infusion fluid, subretinal fluid, blood, and mobile retina float. ${ }^{1}$ So, blood clots and membranes around PFCL can be removed easily by MVP while its cutter port may be kept away from PFCL. As SRF is less dense than PFCL, application of PFCL over posterior pole displace SRF anteriorly to exit subretinal space through retinal breaks (RBs) or retinotomy openings. The settings of laser applying under PFCL should be reduced as SRF is completely gone unlike FAE and internal drainage of SRF. ${ }^{1-9}$

In PFCL assisted IFV, the movement of the retina due to pulsatile vitreoretinal traction (VRT) caused by the cutter opening and closing will be less at the region of the PFO-vitreous-interface (PVI) compared with that in the eye with infusion fluid. Additionally, the retinal tissuewill move more slowly when dissection forces with VRS forceps or scissors were applied duringmembrane peeling/ segmentation/delamination. So, the residual vitreous traction can be easily seen and removed at the PVI. ${ }^{1-9}$

PFO is choice for IFV during the treatment of RD due to particularly with giant RBs or multiple RBs. Full PFO fill is performed for giant $\mathrm{RBs}$, and while as PFO is filled to the posterior border of the most posterior RB for total RDs with multiple RBs. Then, the residual 
vitreous is visualized at PVI, an MVP is placed within vitreous and the removal of residual or peripheral vitreous is performed to maintain a single indwelling PFO bubble. Another option for enhancing the visualization of residual or peripheral vitreous is retro-illumination by reflected light from the top of the PVI. To enhance the visualization of peripheral vitreous may be allowed not only PFO-assisted IFV but also air- or SOIL-assisted IFV..$^{1-9}$

\section{SOIL-assisted IFV}

SOIL allows a half of interfacial surface tension due to air or gas interface with infusion fluid or aqueous humor. Thus, the relative force caused by interfacial surface tension and retinal surface contraction may be properly assessed with SOIL assisted-IFV. ${ }^{1-9}$ The SOIL-vitreous-interface (SVI) provides excellent visualization of residual vitreous and epiretinal membrane. As SOIL has 1,000-5,000 times the viscosity than that of infusion fluid. SOIL allows" viscous dampening" which reduces unwanted retinal movement during ERM peeling, removal of residual vitreous traction, retinectomy, ERM peeling/segmentation/delamination and subretinal surgery by virtue of its high viscosity. SOIL also restricts bleeding into the interface. Above mentioned interventions may be easily performed just outside the SOIL bubble and at the near the retinal surface in SOIL-assisted IFV. Pulsatile VRT produced by the opening and closing of the cutter of MVP is reduced with SOIL-assisted IFV. ${ }^{1-9}$ Intraocular lens (IOL) fogging is an important visualization matter in VRS due to fluid-air exchange (FAE) in the cases having IOL with a YAG capsulotomy. SOIL-assisted IFVresolves this matter. ${ }^{1-10}$ If IOL fogging develops, SOILis injected via the supero-temporal cannula while air exits from the vitreal cavityvia the superonasal cannula. When SOIL reaches the posterior surface of the IOL, the fogging on IOL disappears, and internal drainage of SRF, endo-laser and other interventions for VRSmay be performed "under SOIL". ${ }^{-9}$

During SOIL-assisted IFV, the vacuum should be used at the highest level $(600-650 \mathrm{mmHg})$ and vacuum only applied after the cutter has traversed the SOIL bubble, and the MVP port is at the SVI. Simultaneous retinectomy and removal of SRF are often required in PVR cases, especially for inferior PVR. Residual peripheral VRT can be easily visualized and removed at the SVI..$^{1-9}$ In re-operation under SOIL in case of recurrent RD or removal of ERM without removing SOIL, removal of SRF, residual vitreous and/or the liquid meniscus layer on the retinal surface requires volume replacement with SOIL. This may be achieved with a two-port 25-or 23-gauge technique without the infusion cannula port. ${ }^{1-9}$

\section{Air-assisted IFV}

In air-assisted IFV, air provides a wider field of view and improves visualization. It reduces unwanted retinal movements stabilizing the retina and also restricts bleeding to the air-retinal interface with spring damping effect. This is due to the inherent spring-like properties of compressible gas. Air also prevents infusion fluid from flowing into the subretinal space. Its spring dampening effect is the contrast to the viscous dampening of SOIL and inertial and gravitational stabilization of PFCL. Residual vitreous can sometimes be visualized at the airvitreous interface during fluid-air exchange. ${ }^{1-9}$ In this case, the soft-tip cannula is extracted and, an MVPis re-inserted and the removal of residual vitreous causing to frank VRT is safely performed at the airvitreous interface while FAE continues. This is called "vitrectomy under air" or "air-assisted IFV". ${ }^{1-9}$ Air may sometimes come into contact with the posterior surface of an IOLdue to a posterior capsular defect and the fogging on IOL may occur. In this case, it should be gone back to infusion with the balanced salt solution to eliminate fogging. Retina should be attached with PFCL, endo-laser photocoagulation should be performed on aroundRBs, and PFO-isoexpansive gas exchange using with a soft-tip cannula at the optic cup should be performed.However, vitrectomy under air following FAE in aphakic cases having striate keratopathy or phakic cases having corneal pathologies is well-nigh impossible because of high distortion in fundus view. ${ }^{1-9}$

\section{Conclusion}

Interface vitrectomy allows good intraoperatively stabilization of the retina and the visualization of the vitreous base and vitreoretinal pathologies and it prevents an increase of SRF during traction removal and bleeding to the interface during VRS.

\section{Acknowledgments}

None.

\section{Conflicts of interest}

The authors declare that there is no conflict of interest regarding the publication of this paper and they did not received any fund or financial support for preparation of this paper.

\section{Compliance with ethical guidelines}

This article did not involve any studies with human or animal subjects performed by any of the authors because of the review nature of the article. Authorship: All named authors meet the criteria of the International Committee of Medical Journal Editors (ICMJE) for authorship for this manuscript, take responsibility for the integrity of the work as a whole and have given final approval for the version to be published. All named authors have contributed to the concept and design, data collection, literature Search in the work and writing of the manuscript.

\section{References}

1. Charles S. The Interface Vitrectomy Technique. Retina Today. 2010;50-52.

2. Charles S. Interface vitrectomy. Retin Physician. 2012;9(JanFeb):68-72.

3. Charles S, Randolph JC, Sigler EJ. Pars plana vitrectomy reoperation without removing silicone oil. Retina. 2012;32(8):1664-1665.

4. Charles S, Eliott D, Scott IU. Reoperating without removing silicone oil: Interface vitrectomy. Retina Today. 2008;26-27.

5. Charles S. Vitrectomy techniques for complex retinal detachments. Taiwan J Ophthalmol. 2012;2(3):81-84.

6. Charles S. Management of subretinal fluid and surface tension agents during vitreoretinal surgery. Retin Physician. 2011;64-66.

7. Voleti VB, Gee CJ, Devin F, et al. Vitrectomy under air. Retina. 2012;32(9):1981-1982.

8. Sigler EJ, Charles S, Calzada JI. Interface vitrectomy Retina. 2014;34(3):616-617.

9. Boral SK, Huang SS. Interface Vitrectomy. Retina \& Vitreous Surgery: A Practical Guide to Theory, Practice, and Management. New Delhi: Jaypee Brothers Medical Publishers Ltd; 2016. p. 180-182. 